\title{
Article
}

\section{Grazing Incidence Small-Angle Neutron Scattering: Background Determination and Optimization for Soft Matter Samples}

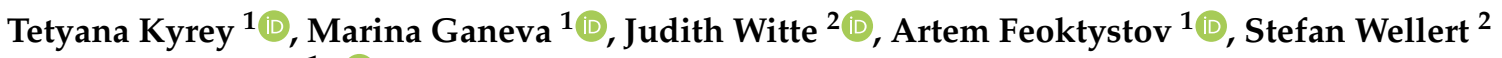 \\ and Olaf Holderer $1, *$ (D) \\ 1 Forschungszentrum Jülich GmbH, Jülich Centre for Neutron Science (JCNS) at Heinz Maier-Leibnitz Zentrum \\ (MLZ), 52425 Garching, Germany; t.kyrey@fz-juelich.de (T.K.); m.ganeva@fz-juelich.de (M.G.); \\ a.feoktystov@fz-juelich.de (A.F.) \\ 2 Institute of Chemistry, Technical University Berlin, 10623 Berlin, Germany; \\ judith.witte@campus.tu-berlin.de (J.W.); s.wellert@tu-berlin.de (S.W.) \\ * Correspondence: o.holderer@fz-juelich.de
}

Citation: Kyrey, T.; Ganeva, M.; Witte, J.; Feoktystov, A.; Wellert, S.; Holderer, O. Grazing Incidence Small-Angle Neutron Scattering: Background Determination and Optimization for Soft Matter Samples. Appl. Sci. 2021, 11, 3085. https:// doi.org/10.3390/app11073085

Academic Editor: Shuo Qian

Received: 25 February 2021

Accepted: 27 March 2021

Published: 30 March 2021

Publisher's Note: MDPI stays neutral with regard to jurisdictional claims in published maps and institutional affiliations.

Copyright: (c) 2021 by the authors. Licensee MDPI, Basel, Switzerland. This article is an open access article distributed under the terms and conditions of the Creative Commons Attribution (CC BY) license (https:// creativecommons.org/licenses/by/ $4.0 /)$.
Featured Application: Scattering contributions in a grazing incidence small-angle neutron scattering experiment come not only from the evanescent wave, but may also come from small-angle scattering of the transmitted beam. Simulations can be used to quantify this contribution. Optimization of the sample cell may reduce an additional background scattering from the cell.

Abstract: Grazing incidence small-angle neutron scattering (GISANS) provides access to interfacial properties, e.g., in soft matter on polymers adsorbed at a solid substrate. Simulations in the frame of the distorted wave Born approximation using the BornAgain software allow to understand and quantify the scattering pattern above and below the sample horizon, in reflection and transmission, respectively. The small-angle scattering from the interfacial layer, visible around the transmitted beam, which might contribute also on the side of the reflected beam, can be understood in this way and be included into the analysis. Background reduction by optimized sample cell design is supported by simulations, paving the way for an optimized GISANS cell.

Keywords: neutron scattering; simulation; GISANS; BornAgain Software; grazing incidence scattering

\section{Introduction}

In the field of soft matter, interface coatings and interface layers consisting of polymers, polymer microgels, brushes or lipid bilayers are very promising in context of practical application in medicine, smart coatings or switching devices [1-4]. However, investigation and characterisation of the interfaces, especially those buried in the sample, are very challenging and can be done by very limited numbers of experimental techniques. Neutron reflectometry allows to study the scattering length density with sub-nanometer resolution in normal direction to the interface [5], while the grazing incidence geometry gives access to the in-plane direction, i.e. to the lateral correlations within the interfacial layers. Grazing incidence small-angle neutron scattering (GISANS) allows a unique access to the interfacial regions, e.g., solid-liquid or liquid-liquid interfaces. Additional information can be obtained by combining grazing incidence neutron scattering and contrast variation via $\mathrm{H}_{2} \mathrm{O} / \mathrm{D}_{2} \mathrm{O}$ mixing, which has been used e.g., in studies of phospholipid bilayers or diblock copolymers [6-8].

Very recently Jaksch and co-authors gave an overview of the field of grazing incidence scattering (GIS) for in-situ and operando investigations in soft matter and biophysics [9]. While GISANS is established at most neutron sources as a technique that gives access to the internal lateral structure of soft matter system at the solid or water/oil interface [9-16], grazing incidence neutron spin echo spectroscopy (GINSES) is a rather novel extension of 
GISANS which allows to study the dynamics of thin polymer films, microemulsions, phospholipid membranes, etc. under similar conditions at the solid/liquid interface [7,17-20].

All these investigations have in common that only a small amount of material contributes to the scattering signal. Therefore for the most precise measurements in this field, background optimisation is of high importance in the experiment [21]. Significant care has to be taken to avoid unnecessary background contributions and to provide a sample environment with long time stability for long experimental times. Such improve of experiments can be done by combining new ways of simulation of the results and according adaption of the experimental conditions [22]. In this case, simulations of the scattering experiments may help (i) to understand the different scattering contributions to the measured signal and (ii) to interpret the experimental data.

In the first part of this paper, we present an example where GISANS experiments together with simulations help to understand the scattering signal and background contributions on the side of reflection and transmission of the GISANS cell. Since scattering under grazing incidence is treated within the distorted wave Born approximation (DWBA) [23], simulations were performed with BornAgain being a modern and easy-to-use open-source software package suitable for simulating GISANS scattering (and also the equivalent $\mathrm{x}$-ray scattering GISAXS) within the DWBA [24,25]. In the second part, we propose a simple version of sample cell for GISANS experiment aiming decrease the background scattering from the cell parts. In both parts, the SANS contribution to the GISANS signal is discussed.

\section{Materials and Methods}

\subsection{Samples of Interest}

PEG-microgels based on the monomer 2-(2-methoxyethoxy)ethyl methacrylate (MEO2MA), the comonomer poly(ethylene glycol) methyl ether methacrylate (OEGMA) and the crosslinker ethylene glycol dimethacrylate (EGDMA) were synthesized by precipitation polymerization. The microgels were deposited on the solid surface by spincoating an aqueous suspensions with a microgel content of $5 \mathrm{wt} \%$ onto the pure silicon block. The microgels of the form $\mathrm{p}$ (MEO2MA-co-OEGMA) were one of our test systems we investigated in the first part of the report. Further details can be found in Ref. [11].

PNIPAM-microgels based on the monomer $N$-isopropylacrylamide (NIPAM) and the cross-linker $N, N^{\prime}$-methylenebisacrylamide (BIS) were synthesised via surfactant-free precipitation polymerisation, with a cross-linker content of $0.5 \mathrm{~mol} \%$. Microgels were deposited onto the PEI-coated silicon blocks via spin coating for $150 \mathrm{~s}$ with $500 \mathrm{rpm}$ from aqueous microgel dispersion. Further details can be found in Ref. [13]. PNIPAM microgels were used in the test of the new self-developed cell.

\subsection{Principles of Grazing Incidence Scattering}

A description of grazing incidence scattering and the distorted wave Born approximation (DWBA) can be found in the literature, e.g., in Refs. [9,23,26]. Here we just mention briefly the main characteristics of a soft matter GISANS experiment.

Typically, soft samples undergo structural changes when adsorbing to a solid substrate. For example, spherical microgel particles can be distorted and compressed when adsorbed at an interface [13]. Studying such soft matter systems consisting of polymer layers of the order of $100 \mathrm{~nm}$ is a challenging task due to the essentially very small scattering volume. Nevertheless, neutron reflectometry and scattering under grazing incidence allows us to obtain valuable information on the scattering length density normal to the surface as well as on lateral (in-plane) correlations at the interface, which correspond to thermal density fluctuations or spacial arrangements with a characteristic length scale [27].

GISANS scattering is normally treated in the DWBA, which takes into account multiple scattering processes at the interface $[25,27,28]$. According to Ref. [29] the diffuse scattered 
intensity (that accompanies the specular reflection) coming from lateral scattering length density fluctuations with intensity:

$$
I(q) \sim\left\langle\left[F_{D W B A}(q)\right]^{2}\right\rangle S(q),
$$

where the term $F_{D W B A}(\mathrm{q})$ is a DWBA form factor accounting for different scattering scenarios with multiple scattering events as described e.g., in Ref. [23].

GISANS is therefore predestined for studying the lateral inhomogeneities. In the case of soft matter samples, lateral structuring is rather blurred and lacking clear Bragg peaks or Bragg sheets, therefore a more detailed modelling of the experiment is needed.

In grazing incidence small-angle scattering (GISAS) experiment incident radiation impinges at a shallow incident angle $\alpha_{i}\left(<1^{\circ}\right)$ and the scattered intensity is detected with a position sensitive detector as a function of scattering $\left(\alpha_{f}\right)$ and out-of-plane $(\psi)$ angles (see Figure 1). The sample surface is the $(x, y)$-plane with the incident neutron beam coming along the $x$-axis, and the scattering plane where the detector is located is the $(y, z)$-plane [30].

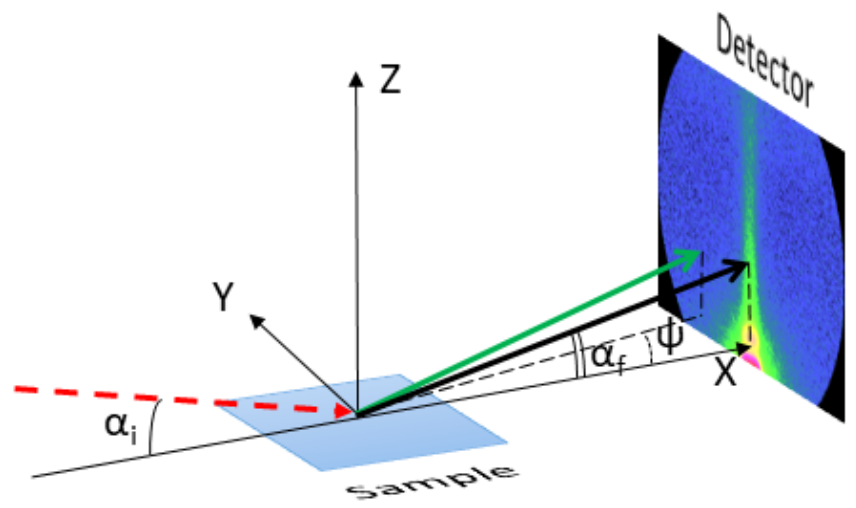

Figure 1. Scheme of grazing incidence scattering experiment. Red dashed and black solid arrows represent incoming and scattered beam, while green solid arrows corresponds to the diffuse scattering. $\alpha_{i}, \alpha_{f}, \psi$-incidence, scattering and out-of-plane angle, respectively.

At $\alpha_{f}=\alpha_{i}$ specular reflection occurs. The interesting part however for GISANS is the off-specular or diffuse scattering, where $\alpha_{f} \neq \alpha_{i}$ [31].

A characteristic part of the detector image in a GISANS experiment is the so-called Yoneda peak [32], which appears at $\alpha_{f}=\alpha_{c}$, with the critical angle of total reflection, $\alpha_{c}$. The cut through the 2D detector pattern at the position of the Yoneda peak provides information on correlations in the lateral density distribution, for example the average inter-particle distance, the size and the shape of the nanostructures in the near surface region $[26,33]$. When $\alpha_{i}>\alpha_{\mathcal{c}}$, the beam is partly reflected and partly refracted, the thin film is then fully illuminated.

If the incidence angle at which the neutron beam hits the sample surface is smaller than the critical angle of total reflection, $\alpha_{i}<\alpha_{c}$, the beam is totally reflected and a specular reflection appears on the detector. However, inside the medium an evanescent intensity distribution with the signature of a propagating wave in $z$-direction parallel to the surface is induced. This "evanescent wave" arises as a real part of the solution of the Schrödinger equation at $\alpha_{i}<\alpha_{c}$ (a mathematical description of the evanescent wave nature can be found elsewhere [34-36]).

The evanescent wave (EW) probes the sample only to a short distance from the probed interface (up to $100 \mathrm{~nm}$ ) [16]. The intensity of the EW drops exponentially with the distance.

In this work, we used the software BornAgain [25] to mimic a GISANS experiment as close as possible with its 2D detector image for a given incident angle. The sample, in this case microgel particles adsorbed at the silicon interface, was modelled as core-shell truncated spheres with pre-knowledge from neutron reflectometry and AFM measure- 
ments [24]. The scattering signal was then obtained on small-angle neutron scattering instruments in grazing incidence geometry.

\subsection{Grazing Incidence Small-Angle Scattering Experiment}

GISANS experiments were performed on KWS-1 [37,38] and KWS-2 [39,40] smallangle neutron scattering instruments operated by Jülich Centre for Neutron Science at Maier-Leibnitz Zentrum (Garching, Germany).

Measurements on KWS-1 were performed at a sample-to-detector distance of $20 \mathrm{~m}$ using an unpolarised, monochromatic incident beam with a neutron wavelength of $5 \AA$ $(\Delta \lambda / \lambda=10 \%)$ under an angle of incidence $\left(\alpha_{i}\right)$ of $0.2^{\circ}$. Experiments on KWS-2 were conducted at sample-to-detector distances of $4 \mathrm{~m}$ with a neutron wavelength of $5 \AA(\Delta \lambda / \lambda=20 \%)$ and under an angle of incidence $\left(\alpha_{i}\right)$ of $0.7^{\circ}$. This angle slightly above the critical angle was chosen to probe the structure of the entire layer of microgel particles.

In GISANS experiments samples were mounted in cells developed for grazing incidence geometry: PEG microgels in aluminium cell [41] and PNIPAM microgels in self-made quartz cell. Both cells were filled with $\mathrm{D}_{2} \mathrm{O}$ providing high scattering contrast to the polymers and low incoherent scattering.

\section{Results and Discussion}

\subsection{Transmission Contribution to GIS Pattern}

GISANS experiments together with the corresponding simulations with BornAgain were performed on samples of PEG-microgels adsorbed at a silicon interface and are presented in Figure 2. The same GISANS data have been presented in Ref. [11], with a preliminary evaluation of the line cuts which did not cover all details of the experimental data. The angle of incidence $\alpha_{i}=0.7^{\circ}$ at a wavelength of $5 \AA$ slightly above the critical angle of total reflection has been chosen to strongly illuminate the microgels.

(a)

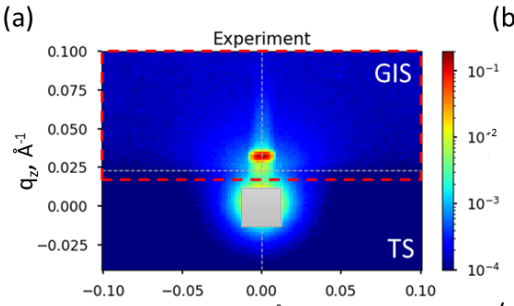

(b)

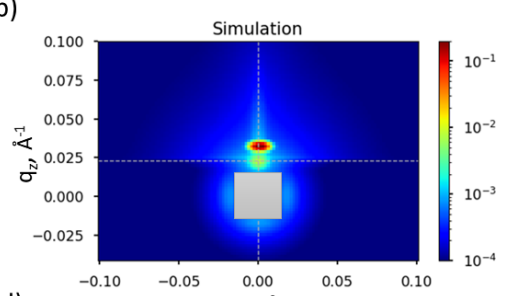

(d)
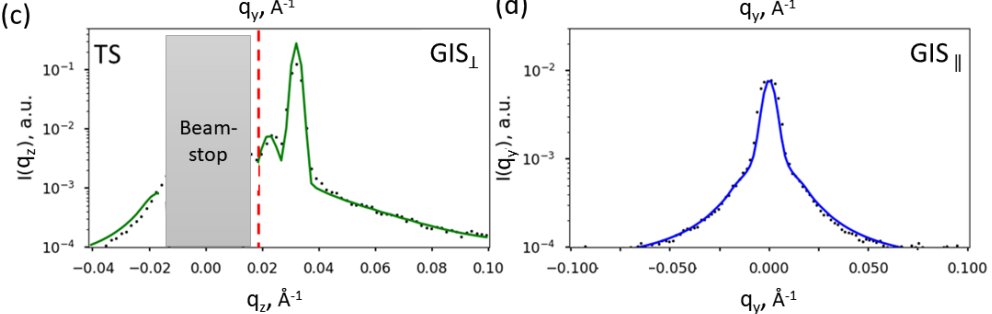

Figure 2. Experimental (a) and simulated with BornAgain (b) 2D grazing incidence small-angle neutron scattering (GISANS) scattering pattern of adsorbed p(MEO2MA-co-OEGMA) microgels. White dashed lines in $(\mathbf{a}, \mathbf{b})$ correspond to the cuts depicted in $(\mathbf{c}, \mathbf{d})$. In $(\mathbf{c}, \mathbf{d})$ the perpendicular (along $q_{x}$ ) and the parallel (along $q_{y}$ ) to the sample surface cuts are presented, dots and solid lines in (c,d) correspond to experimental and simulated data, respectively. Grey masks in $(\mathbf{a}-\mathbf{c})$ is beam-stop that blocks direct beam. The red dashed line in (c) represents the sample horizon.

The analysis of 2D GISANS detector images is mainly focused on the part containing the totally reflected beam above the sample horizon, whereas the part below the sample horizon, where the transmitted beam and small angle scattering still reaches the detector, is often ignored. A first analysis of the experiments on PEG-microgels at the interface was done in this way (as presented in Ref. [11]). 
If the incident angle $\alpha_{i}$ was slightly above the critical angle of total reflection $\left(\alpha_{c}\right)$ as in case of PEG-microgels, transmission scattering (TS) occurred in addition to the specular and diffusive signal. Therefore, in the following analysis of the scattering 2D pattern, it was recognized that the transmitted scattering may in particular also have extended into the detector half of the GIS signal, contributing to the diffuse scattering as a background.

The transmission signal was reconstructed with BornAgain by applying the PEGmicrogel model [26], and included into the GIS simulation. The scattering below the sample horizon in the TS part corresponded to the scattering of a transmission experiment at the surface layer with a slightly inclined beam. Thus, the incident angle $\alpha_{i}$ was set to $90^{\circ}$ for the transmission scattering simulation. A direct calculation in grazing incidence geometry would be more appropriate, but is at the moment not yet implemented in the BornAgain software. Accounting for the SANS signal in this way well explained in our case the scattering contributions of the GISANS pattern and seemed to be adequate in this case. It should be noted, that such a sample rotation in virtual experiment is possible in case of an isotropic system in the probed length-scale. If structural anisotropy at the considered length scale comes into play, care has to be taken concerning details of the transmission scattering pattern.

In contrast to the highly collimated and monochromatic beam of grazing incidence small angle X-ray scattering experiments, GISANS experiments normally use a neutron beam with a much broader width and a wavelength distribution of $10-20 \%$ in order to obtain enough intensity. This leads to some peculiarities, which should not be overlooked during the data treatment and simulations.

The influence of the beam divergence on the scattering volume of the sample and on the footprint of the beam on the detector is depicted in Figure 3. The difference in scattering volume of areas 1 and 2 corresponded to the different scattering intensity on the $2 \mathrm{D}$ scattering pattern in the regions $\mathrm{AB}$ and $\mathrm{BC}$. Angles are expanded in Figure 3 compared to a real experiment.

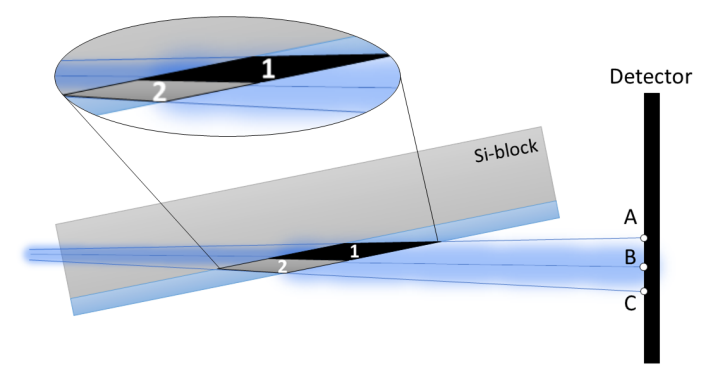

Figure 3. Schematic illustration of the transmission in grazing incidence geometry: difference in the footprints of the upper- and lower- beam parts due to the beam divergence.

The difference in the intensity distribution in area $\mathrm{AB}$ and $\mathrm{BC}$ (Figure 3) was taken into account in the following way. A matrix of the 2D scattering signal was multiplied with a vector, which provided more intense scattering in the upper part of the TS pattern (intensity ratio of 2.5:1 was taken, which depended on the scattering geometry and beam size). Simulating the lower transmission part is also a means of judging possible scattering contribution from the environment and the sample cell. If a simulated GIS pattern differs from the experimental observation additional contributions to the background should be considered.

Figure 2 shows the experimental and the simulated 2D GISANS signal including the contribution of the transmitted scattering in the direct beam direction [26]. The simulated 2D pattern as well as line cuts at $q_{y}=0$ (along $q_{z}$ ) and at $\alpha_{c}$ (parallel to the sample surface) were in good agreement with the experimental data. There are still some remaining differences in the diffuse scattering at the borders of the upper detector part (Figure 2a,b), which might be attributed to the instrumental effects not included into the BornAgain simulation, or sample effects such as the particle size distribution, which were also not part 
of the simulation. The chosen beam parameters allowed an estimation of lateral correlation length, while resolution in the direction normal to the surface led to some crossing over of specular and Yoneda peak. Here it should also be noted that reproduction of profile of direct beam, such as simulation of the beam collimation, angular divergence etc., is a difficult task and is not too relevant in this case. We focus more on regions of large $q$, and diffuse scattering fluctuations, while the beam profile could have a stronger influence on low- $q$ scattering or Bragg-peaks. This is still to be improved for future investigation of larger real space distances.

\subsection{Test of Self-Made Cell with Reduced Background}

With the previous observation that the background coming from the small-angle scattering of the sample might play a role and that also the side of the transmitted beam might influence a proper data evaluation, a new GISANS cell design with minimized material around the sample was tested. PNIPAM microgels adsorbed on Si-block were used as a test sample.

Figure 4 shows a photograph of the self-made quartz cell (a), its components (b) and the principal beam path for reflection and transmission. Basically, a quartz glass backplate was placed on a frame with boron containing glass for neutron absorption, both were directly glued together with the Si-block which contained at its surface the sample (PNIPAM-microgel particles). Small gaps on one side with syringe needles allowed us to fill the cell with water. The borated glass was used to reduce possible scattering by the glue at the interface between the glass layers. What is the optimum condition and material in this respect has to be studied in more detail in the future. $\mathrm{D}_{2} \mathrm{O}$ was used to increase scattering contrast to the PNIPAM microgels and avoid high incoherent contribution as could be in case of $\mathrm{H}_{2} \mathrm{O}$.

a)

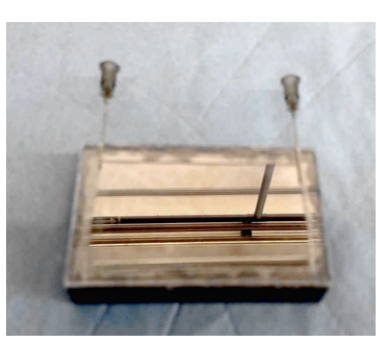

b)

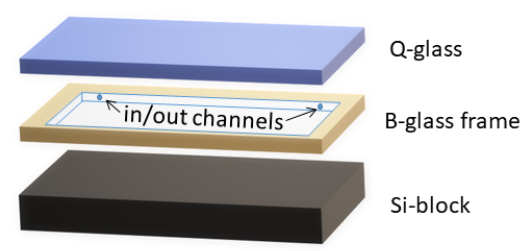

c)

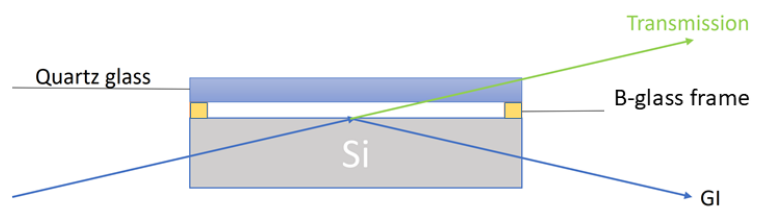

Figure 4. Cell design with minimum amount of auxiliary material. (a) shows a photograph of the cell, the assembly is presented in $(\mathbf{b})$. The beam path for reflected and transmitted beam is shown in (c).

This cell design with its minimum surrounding opens the new possibility of measuring the dynamics of polymer systems in grazing incidence geometry by means of grazing incidence neutron spin echo spectroscopy (GINSES). Due to the instrumental setup of neutron spin echo spectrometer and very limited area for the in- and out-path for the beam usually used in an aluminium cell, currently GINSES can only be measured off-specular in $q_{z}$ direction at $q_{y}=0$. With proposed cell design it would be possible to rotate the sample cell for a scan in the direction of $q_{y}$, giving access to lateral dynamics along the interface.

The first GISANS experiment with the proposed cell is presented in Figure 5. The left side of the detector image contained the part with the reflected beam and the GISANS scattering. On the right side of the detector (within green frame) the transmitted beam was visible. The line cut along the $q_{z}$ direction at $q_{y}=0$ is presented on the right side of Figure 5 . 

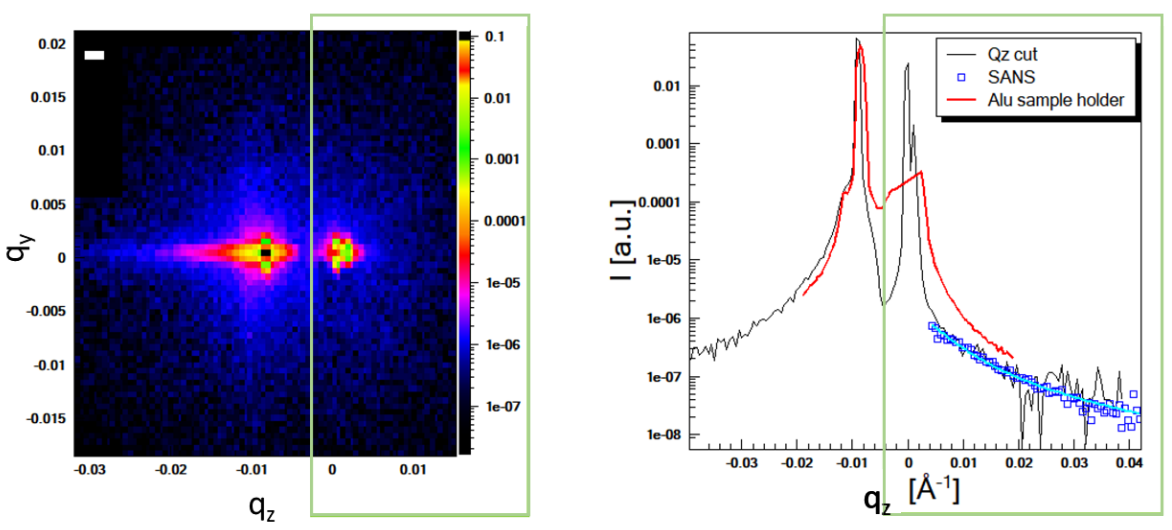

Figure 5. Left: Detector image of microgels at interfaces with the new cell: the GISANS part (left side of the detector) and the SANS part around the transmitted beam (within green frame). Right: Line cuts of the detector images along $q_{z}$ at $q_{y}=0$ (black line), compared to the data measured with Al cell (red line). A significantly reduced background signal is obtained. The radially averaged SANS intensity of the right side of the detector is shown as blue squares with a fit with an OrnsteinZernike contribution.

The line cuts of the data obtained with our cell with minimal material (black line) were compared to that of the often used design with aluminum front- and back-plates with water channels for thermalization (red line). On the transmission side, additionally the radially averaged SANS intensity of this side of the detector is presented (blue dots) with a fit of the data (cyan) with an Ornstein-Zernike model for thermal fluctuations [13,42].

A significantly reduced background could be achieved with the proposed new design, which was necessary for the low-intensity GISANS experiments with biological or soft matter systems and allowed us to focus only on the scattering of the desired parts of the sample.

The self-made cell had the advantage that only a minimum amount of cell material was required, with the disadvantage that the temperature control was not possible with such a cell in the usual way done with $\mathrm{Al}$ cell and had to be provided by other means. For example, a thermalized air jet in the sample area, as it is used also for other "conventional" experiments, e.g., in Ref. [43] for the thermal control of an in-situ DLS setup.

\section{Conclusions}

The low intensity of typical samples in GISANS experiments in the field of soft matter and biology requires thorough experimental planning, data reduction and data interpretation. On the experimental side, the sample cell is a crucial part of the experiment. It has been shown that not only the side of the detector containing the reflected beam and the GISANS data, but also the backside with the transmitted beam contains valuable information and helps understanding different contributions to the signal and to the background. The small-angle scattering signal, i.e., the form factor of the monolayer of microgels present around the transmitted beam, contributed in the current experiment to a background which is not flat. The evaluation in Ref. [11] has been done without such a contribution and showed a significant deviation between measured data and optimal fitting curve with an Ornstein-Zernike model. The gain provided by simulating this SANS contribution with BornAgain provides a means of taking this into account for the interpretation of the GISANS images. Taking into account this additional background which also extends into the upper half of the detector above the horizon of the scattering plane is important if low intensities are evaluated. Soft matter GISANS experiments suffer in many cases from low intensity and rather blurred features on the detector, making this kind of analysis necessary.

The BornAgain simulation software is a very valuable tool for assessing the background contributions. This has been shown here for GISANS of microgel particles at the 
solid interface, and also helped in understanding background contributions for GINSES experiments (see Ref. [24]).

Testing a sample cell design with very little amount of material for GISANS showed very promising results in terms of a reduced background from the sample cell. This design would in principle also allow for new scan mode for GINSES experiments in the $q_{y}$ plane to study dynamics, e.g., diffusive processes parallel to the interface.

Grazing Incidence Neutron Scattering provides unique information on the structure and also on dynamics at interfaces. It is a challenging work to study such small amounts of material as it is common for soft matter samples, but if the necessary care is taken concerning experimental background considerations and parallel simulation of the experiment, valuable information on the interface structure can be obtained.

Author Contributions: conceptualization, T.K., O.H., S.W., J.W., A.F. methodology, O.H.; software, M.G.; formal analysis, T.K.; writing-original draft preparation, O.H., T.K.; writing-review and editing, S.W. All authors have read and agreed to the published version of the manuscript.

Funding: This research was funded by Deutsche Forschungsgemeinschaft (DFG) (grant Nos. HO 5488/2-1 (OH) and WE 5066/3-1 (SW)).

Data Availability Statement: Data presented in this study can be obtained from the authors upon request.

Acknowledgments: The authors gratefully acknowledge the financial support provided by JCNS to perform the neutron scattering measurements on the KWS-1 and KWS-2 instruments at the Heinz Maier-Leibnitz Zentrum (MLZ), Garching, Germany. Fruitful discussion with Henrich Frielinghaus is acknowledged.

Conflicts of Interest: The authors declare no conflict of interest.

\author{
Abbreviations \\ The following abbreviations are used in this manuscript: \\ MDPI Multidisciplinary Digital Publishing Institute \\ DOAJ Directory of open access journals \\ TLA Three letter acronym \\ LD Linear dichroism
}

\title{
References
}

1. Gao, Y.; Serpe, M.J. Light-Induced Color Changes of Microgel-Based Etalons. ACS Appl. Mater. Interfaces 2014, 6, 8461-8466. [CrossRef]

2. Nolan, C.M.; Serpe, M.J.; Lyon, L.A. Pulsatile Release of Insulin from Layer-by-Layer Assembled Microgel Thin Films. Macromol. Symp. 2005, 227, 285-294. [CrossRef]

3. Uhlig, K.; Wegener, T.; He, J.; Zeiser, M.; Bookhold, J.; Dewald, I.; Godino, N.; Jaeger, M.; Hellweg, T.; Fery, A.; Duschl, C. Patterned Thermoresponsive Microgel Coatings for Noninvasive Processing of Adherent Cells. Biomacromolecules 2016, 17, 1110-1116. [CrossRef]

4. Cichosz, S.; Masek, A.; Zaborski, M. Polymer-based sensors: A review. Polym. Test. 2018, 67, 342-348. 2018.03.024. [CrossRef]

5. Majkrzak, C.F.; Berk, N.F.; Krueger, S.; Dura, J.A.; Tarek, M.; Tobias, D.; Silin, V.; Meuse, C.W.; Woodward, J.; Plant, A.L. First-principles determination of hybrid bilayer membrane structure by phase-sensitive neutron reflectometry. Biophys. J. 2000, 79, 3330-3340. [CrossRef]

6. Mangiapia, G.; Gvaramia, M.; Kuhrts, L.; Teixeira, J.; Koutsioubas, A.; Soltwedel, O.; Frielinghaus, H. Effect of benzocaine and propranolol on phospholipid-based bilayers. Phys. Chem. Chem. Phys. 2017, 19, 32057-32071. [CrossRef]

7. Jaksch, S.; Lipfert, F.; Koutsioumpas, A.; Mattauch, S.; Holderer, O.; Ivanova, O.; Frielinghaus, H.; Hertrich, S.; Fischer, S.F.; Nickel, B. Influence of ibuprofen on phospholipid membranes. Phys. Rev. E 2015, 91, 022716. [CrossRef]

8. Müller-Buschbaum, P.; Cubitt, R.; Petry, W. Nanostructured Diblock Copolymer Films: A Grazing Incidence Small-Angle Neutron Scattering Study. Langmuir 2003, 19, 7778-7782. [CrossRef]

9. Jaksch, S.; Gutberlet, T.; Müller-Buschbaum, P. Grazing incidence scattering - status and perspectives in soft matter and biophysics. Curr. Opin. Colloid Interface Sci. 2019, 42, 73-86. [CrossRef]

10. Nouhi, S.; Hellsing, M.S.; Kapaklis, V.; Rennie, A.R. Grazing-incidence small-angle neutron scattering from structures below an interface. J. Appl. Crystallogr. 2017, 50, 1066-1074. [CrossRef] 
11. Kyrey, T.; Ganeva, M.; Gawlitza, K.; Witte, J.; von Klitzing, R.; Soltwedel, O.; Di, Z.; Wellert, S.; Holderer, O. Grazing incidence SANS and reflectometry combined with simulation of adsorbed microgel particles. Phys. B Phys. Condens. Matter 2018, 551, 172-178. [CrossRef]

12. Müller-Buschbaum, P. The active layer morphology of organic solar cells probed with grazing incidence scattering techniques. Adv. Mater. 2014, 26, 7692-7709. [CrossRef] [PubMed]

13. Kyrey, T.; Witte, J.; Pipich, V.; Feoktystov, A.; Koutsioubas, A.; Vezhlev, E.; Frielinghaus, H.; von Klitzing, R.; Wellert, S.; Holderer, O. Influence of the cross-linker content on adsorbed functionalised microgel coatings. Polymer 2019, 169, 29-35. [CrossRef]

14. Müller-Buschbaum, P.; Wolkenhauer, M.; Wunnicke, O.; Stamm, M.; Cubitt, R.; Petry, W. Structure formation in two-dimensionally confined diblock copolymer films. Langmuir 2001, 17, 5567-5575. [CrossRef]

15. Wellert, S.; Richter, M.; Hellweg, T.; von Klitzing, R. Responsive Microgels at Surfaces and Interfaces. Z. Phys. Chem. 2014, 229, 1225-1250. [CrossRef]

16. Müller-Buschbaum, P. GISAXS and GISANS as metrology technique for understanding the 3D morphology of block copolymer thin films. Eur. Poly. J. 2016, 81, 470-493. [CrossRef]

17. Nylander, T.; Soltwedel, O.; Ganeva, M.; Hirst, C.; Holdaway, J.; Arteta, M.Y.; Wadsa, M. Relationship between structure and fluctuations of lipid nonlamellar phases deposited at the solid-liquid interface. J. Phys. Chem. B 2017, 121, 2705-2711. [CrossRef]

18. Lipfert, F.; Frielinghaus, H.; Holderer, O.; Mattauch, S.; Monkenbusch, M.; Arend, N.; Richter, D. Polymer enrichment decelerates surfactant membranes near interfaces. Phys. Rev. E 2014, 89, 042303. [CrossRef] [PubMed]

19. Frielinghaus, H.; Kerscher, M.; Holderer, O.; Monkenbusch, M.; Richter, D. Acceleration of membrane dynamics adjacent to a wall. Phys. Rev. E 2012, 85, 041408. [CrossRef]

20. Gawlitza, K.; Ivanova, O.; Radulescu, A.; Holderer, O.; von Klitzing, R.; Wellert, S. Bulk phase and surface dynamics of PEG microgel particles. Macromolecules 2015, 48, 5807-5815. [CrossRef]

21. Hoogerheide, D.P.; Heinrich, F.; Maranville, B.B.; Majkrzak, C.F. Accurate background correction in neutron reflectometry studies of soft condensed matter films in contact with fluid reservoirs. J. Appl. Crystallogr. 2020, 53, 15-26. [CrossRef]

22. Treece, B.W.; Kienzle, P.A.; Hoogerheide, D.P.; Majkrzak, C.F.; Lösche, M.; Heinrich, F. Optimization of reflectometry experiments using information theory. J. Appl. Crystallogr. 2019, 52, 47-59. [CrossRef] [PubMed]

23. Lazzari, R. IsGISAXS: a program for grazing-incidence small-angle X-ray scattering analysis of supported islands. J. Appl. Crystallogr. 2002, 35, 406-421. [CrossRef]

24. Kyrey, T.; Ganeva, M.; Witte, J.; von Klitzing, R.; Wellert, S.; Holderer, O. Understanding near-surface polymer dynamics by a combination of grazing-incidence neutron scattering and virtual experiments. J. Appl. Crystallogr. 2021, 54. [CrossRef]

25. Pospelov, G.; Van Herck, W.; Burle, J.; Carmona Loaiza, J.M.; Durniak, C.; Fisher, J.M.; Ganeva, M.; Yurov, D.; Wuttke, J. BornAgain: software for simulating and fitting grazing-incidence small-angle scattering. J. Appl. Crystallogr. 2020, 53, 262-276. [CrossRef] [PubMed]

26. Kyrey, T. Internal Structure and Dynamics of PNIPAM Based Microgels in Bulk and Adsorbed State at Different Internal Crosslinker Distributions. Ph.D. Thesis, Technische Universität, Darmstadt, Germany, 2019.

27. Müller-Buschbaum, P. Grazing incidence small-angle neutron scattering: challenges and possibilities. Poly. J. 2013, 45, 34-42. [CrossRef]

28. Korolkov, D. Structural Analysis of Diblock Copolymer Nanotemplates Using Grazing Incidence Scattering; Forschungszentrum Jülich: Jülich, Germany, 2008.

29. Santoro, G.; Yu, S. Grazing incidence Small Angle X-ray Scattering as a Tool for In-Situ Time-Resolved Studies; In X-ray Scattering; Ares, A.E., Ed.; INTECH: London, UK, 2017; Chapter 2, pp. 29-60.

30. Müller-Buschbaum, P.; Gutmann, P.; Stamm, M.; Cubitt, R.; Cunis, S.; von Krosigk, G.; Gehrke, G.; Petry, W. Dewetting of thin polymer blend films: Examined with GISAS. Phys. B 2000, 283, 53-59. [CrossRef]

31. Lauter-Pasyuk, V. Neutron grazing incidence techniques for nano-science. Collect. SFN 2007, 7, 221-240. [CrossRef]

32. Yoneda, Y. Anomalous surface reflection of X rays. Phys. Rev. 1963, 131, 2010-2013. [CrossRef]

33. Schwartzkopf, M.; Roth, S.V. Investigating polymer-metal interfaces by grazing incidence small-angle X-ray scattering from gradients to real-time studies. Nanomaterials 2016, 6, 239. [CrossRef] [PubMed]

34. Milosevic, M. On the nature of the evanescent wave. Appl. Spectrosc. 2013, 67, 126-131. [CrossRef]

35. Knoll, W. Polymer thin films and interfaces characterized with evanescent light. Die Makromolekulare Chemie 1991, 192, 2827-2856. [CrossRef]

36. Dosch, H. Critical Phenomena at Surfaces and Interfaces; Springer Tracts in Modern Physics: Berlin/Heidelberg, Germany, 1992.

37. Feoktystov, A.V.; Frielinghaus, H.; Di, Z.; Jaksch, S.; Kleines, H.; Ioffe, A.; Richter, D. KWS-1 high-resolution small-angle neutron scattering instrument at JCNS: current state. Appl. Crystallogr. 2015, 48, 61-70. [CrossRef]

38. Zentrum, H.M.L. KWS-1: Small-angle scattering diffractometer. J. Large-Scale Res. Facil. 2015, 1, A28.

39. Zentrum, H.M.L. KWS-2: Small angle scattering diffractometer. J. Large-Scale Res. Facil. 2015, 1, A29. [CrossRef]

40. Radulescu, A.; Pipich, V.; Frielinghaus, H.; Appavou, M.-S. KWS-2, the high intensity/wide Q-range small-angle neutron diffractometer for soft-matter and biology at FRM II. J. Phys. Conf. Ser. 2012, 351, 012026. [CrossRef]

41. Steitz, R.; Gutberlet, T.; Hauss, T.; Klo, B.; Krastev, R.; Schemmel, S.; Simonsen, A.C.; Findenegg, G.H. Nanobubbles and their precursor layer at the interface of water against a hydrophobic substrate. Langmuir 2003, 19, 2409-2418. [CrossRef] 
42. Shibayama, M. Small-angle neutron scattering on polymer gels: phase behavior, inhomogeneities and deformation mechanisms. Polym. J. 2011, 43, 18-34. [CrossRef]

43. Balacescu, L.; Vögl, F.; Staringer, S.; Ossovyi, V.; Brandl, G.; Lumma, N.; Feilbach, H.; Holderer, O.; Pasini, S.; Stadler, A.; et al. In situ dynamic light scattering complementing neutron spin echo measurements on protein samples. J. Surf. Investig. X-ray Synchrotron Neutron Tech. 2020, 14, S185-S189. [CrossRef] 